\title{
ARTÍCULOS
}

\section{COMUNICACIÓN DE DESTINOS TURÍSTICOS A TRAVÉS DE LOS MEDIOS SOCIALES}

\author{
Assumpció Huertas, Dolors Setó-Pàmies y María-Isabel Míguez-González
}

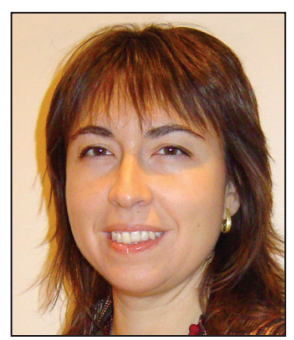

Assumpció Huertas es profesora en el Departamento de Estudios de Comunicación de la Universidad Rovira y Virgili, Tarragona, España, y miembro del grupo de investigación Asterisc de la misma universidad. Es la investigadora principal del proyecto Uso e influencia de los social media y la comunicación 2.0 en la toma de decisiones turísticas y en la imagen de marca de los destinos. Aplicaciones de utilidad para los destinos turísticos españoles (CSO2012-34824); y autora del libro La comunicación de los territorios, los destinos y sus marcas. http://orcid.org/0000-0001-6684-4220

Universidad Rovira i Virgili. Facultad de Letras, Departamento de Estudios de Comunicación Av. Catalunya, 35 (Campus Centre). 43002 Tarragona, España sunsi.huertas@gmail.com

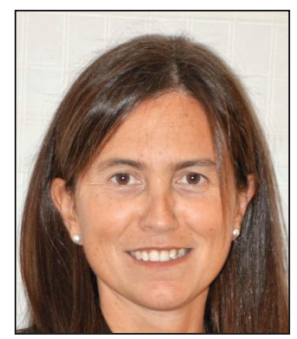

Dolors Setó-Pàmies, doctora en administración y dirección de empresas por la Universitat Rovira $i$ Virgili (URV), es profesora titular de universidad del área de Organización de Empresas, en el Departamento de Gestión de Empresas de la URV. Imparte docencia en la Facultad de Economía y Empresa así como en la Facultad de Turismo y Geografía. Es investigadora en temas de management, calidad y relación con los clientes. Ha sido responsable de la titulación de Administración y Dirección de Empresas (ADE) en la Facultad de Economía y Empresa de la URV. http://orcid.org/0000-0002-8525-6712

Universidad Rovira i Virgili. Facultad de Economía y Empresa, Departamento de Gestión de Empresas Av. de la Universitat, 1. 43204 Reus (Tarragona), España dolors.seto@urv.cat

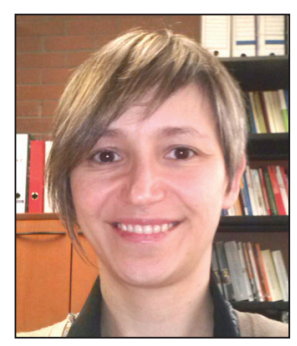

María-Isabel Míguez-González es profesora contratada doctora en la Facultad de Ciencias Sociales y de la Comunicación de la Universidad de Vigo, coordinadora del grado en Publicidad y Relaciones Públicas y docente de teoría y práctica de las relaciones públicas y dirección de comunicación. Investiga en relaciones públicas, turismo y neurocomunicación. Participa en dos proyectos del plan nacional de I+D sobre turismo, web 2.0 y redes sociales. Autora del libro Los públicos en las relaciones públicas (2010).

http://orcid.org/0000-0002-0580-8493

Universidad de Vigo Facultade de Ciencias Sociais e da Comunicación, Departamento de Comunicación Audiovisual y Publicidad Campus A Xunqueira, s/n. 36005 Pontevedra, España mabelm@uvigo.es

\section{Resumen}

Se ofrecen recomendaciones para mejorar el modo en que los destinos turísticos emplean las redes sociales en su comunicación. Mediante herramientas de medición online se analizan las cuentas de Facebook y Twitter de 5 destinos turísticos españoles. Se comprueba que los destinos descuidan en las redes la construcción de la identidad de marca-lugar y que el potencial interactivo se desaprovecha en gran medida, sobre todo en Facebook. Se recomienda potenciar el engagement (compromiso, fidelidad) realizando preguntas a los usuarios y utilizando fotografías, así como incidir en la comunicación de marca y no sólo en la difusión de información turística.

\section{Palabras clave}

Medios sociales; Redes sociales; Facebook; Twitter; Comunicación; Destinos turísticos; Metodología de análisis; Herramientas online de medición.

Title: Social media communications strategies of tourist destinations 


\begin{abstract}
In order to provide recommendations for improving the way in which destinations use social networks in their communication strategy, Facebook and Twitter accounts of important Spanish tourist destinations were analysed, using online measurement tools. The results show that these destinations neglect to build a place-identity brand on the social networks and that interactive potential is largely wasted, especially in Facebook. The recommendation is to enhance user engagement by posing questions to them or posting photos, as well as focusing on brand communication, not only on disseminating tourist information.
\end{abstract}

\title{
Keywords
}

Social media; Social networks; Facebook; Twitter; Communication; Tourist destinations; Analysis methodology; Online measuring tools.

Huertas, Assumpció; Setó-Pàmies, Dolors; Míguez-González, María-Isabel (2015). "Comunicación de destinos turísticos a través de los medios sociales". El profesional de la información, enero-febrero, v. 24, n. 1, pp. 15-21.

http://dx.doi.org/10.3145/epi.2015.ene.02

\section{Introducción ${ }^{1}$}

Con más de mil millones de personas que acceden a internet cada día, los medios sociales se han convertido en una plataforma para el cambio social (Agarwal; Mondal; Nath, 2011). El desarrollo de la web 1.0 hacia la web 2.0 ha sido remarcable, ya que ha permitido no sólo que las personas lean y busquen información sino también que puedan crear y compartir información entre ellas (Berthon; Pitt; Plangger; Shapiro, 2012).

Según InternetWorldStats (2012) el número estimado de usuarios de internet a nivel mundial a finales de 2013 era de 2.800 millones. Esos usuarios gastaron el 22,5\% de su tiempo en redes sociales como Twitter, Facebook y YouTube (Pring, 2012).

Las nuevas tecnologías de la información y la comunicación juegan un papel clave en el desarrollo de los destinos turísticos; teniendo un impacto considerable en la comunicación de su marca y su identidad (Munar, 2011). Los medios sociales permiten a los destinos relacionarse en cualquier momento y directamente con sus distintos públicos a un coste relativamente bajo (Kaplan; Haenlein, 2010). Sin embargo, la gestión de los medios sociales no es una tarea fácil y requiere nuevas formas y maneras de pensar la comunicación (Kaplan; Haenlein, 2010; Agarwal et al., 2011).

Son muchos los estudios existentes en la actualidad sobre el impacto de los medios sociales en turismo, aunque no todos les conceden la misma relevancia. Sigala (2009) o Xian y Gretzel (2010) destacan su importante papel en la selección de destinos por parte de los turistas y Leung et al. (2013) destacan que los viajeros han adoptado herramientas colaborativas como los blogs y microblogs, las comunidades online, las plataformas para compartir vídeos o imágenes o las webs de marcadores sociales para buscar información. Sin embargo, otros estudios (Cox et al., 2009; World Travel Market, 2010), tal y como recogen Ayeh et al. (2013), sugieren que la mayoría de los usuarios de internet no emplean los medios con comentarios generados por los consumidores para organizar sus viajes. En una línea similar Jacobssen y Munar (2012) destacan que, si bien la web social se ha convertido en una fuente complementaria de información para el turista, otras fuentes tradicionales continúan siendo prioritarias.
Por otra parte, muy pocas investigaciones analizan el uso de los medios sociales por parte de los destinos turísticos, y también existe escaso conocimiento sobre su influencia en la comunicación de la marca de un destino turístico (Munar, 2011), aunque parece claro que sí puede influir en la imagen del destino que se genera en la mente del usuario.

Así mismo, diversos estudios (Murphy; Moscardo; Benckendorff, 2007; Litvin et al., 2008; Fotis; Buhalis; Rossides, 2012; Leung et al., 2013) corroboran que los contenidos generados por los usuarios a través de los medios sociales tienden a percibirse como una información más fiable que la ofrecida por otras fuentes más institucionales como las webs oficiales. Por ello, los destinos han empezado a utilizar los medios sociales.

Sin embargo, en muchas ocasiones, debido a la novedad y el desconocimiento de los medios sociales, los destinos turísticos los utilizan sin seguir una planificación estratégica (Hvass y Munar, 2012), sin sacar provecho de todas sus posibilidades, sin saber el uso que los públicos hacen de ellos y sin conocer los beneficios que les pueden aportar para la comunicación de su marca. Su uso entre las organizaciones de marketing de destino es, en gran medida, experimental, y sus estrategias varían de forma significativa (Hays; Page; Buhalis, 2013).

Así pues, el objetivo de este estudio es analizar el uso que los destinos turísticos hacen de los medios sociales como sistemas de comunicación con la finalidad de ofrecer recomendaciones prácticas que sean de utilidad para su mejora.

\section{Metodología}

Se han analizado las cuentas oficiales de Facebook y Twitter de 5 destinos turísticos, las capitales de las cinco comunidades autónomas españolas que corresponden a las áreas Nielsen²:

- Santiago de Compostela
http://www.santiagoturismo.com
- Barcelona
http://www.barcelonaturisme.com
- Sevilla
http://www.visitasevilla.es




\section{- Madrid http://www.esmadrid.com \\ - Las Palmas http://www.lpavisit.com/es}

Detrás de las cuentas de Facebook y Twitter analizadas, a las que se puede acceder desde las webs de los destinos, se encuentran las siguientes entidades: Santiago Turismo (que pertenece al Concello de Santiago de Compostela), Turisme de Barcelona (consorcio dedicado a la promoción turística), Consorcio Turismo de Sevilla, el Área de las Artes, Deporte y Turismo del Ayuntamiento de Madrid, y el Ayuntamiento de Las Palmas de Gran Canaria, desde donde operan los gestores de estos medios sociales.

Teniendo en cuenta que uno de los principales objetivos del proyecto es elaborar una metodología de análisis de los medios sociales, se ha comenzado por establecer los parámetros e items de estudio. Estos parámetros son: contenidos, interactividad y visibilidad.

En contenidos se ha analizado lo siguiente:

- frecuencia de los posts;

- temática;

- tipo de información que suministran (queja, comentario, pregunta, sugerencia); y

- formato que utilizan (texto, fotografías, vídeos).

En interactividad:

- reacciones de los usuarios y su engagement (compromiso o fidelidad).

En visibilidad:

- número de fans o seguidores;

- me gusta;

- comparticiones (shares); y

- favoritos.

Algunas de esas métricas se encuentran en dos parámetros. Por ejemplo, los "me gusta", los comentarios y las comparticiones se miden en el engagement $\mathrm{y}$, por tanto, también se incluyen en el parámetro de la interactividad. Pero dado que son un aspecto clave que mide la visibilidad, también están presentes en éste.

Como la mayoría de variables pueden medirse cuantitativamente mediante numerosas herramientas online, se optó por utilizar algunas de ellas y evaluar así su eficacia. Para seleccionarlas se realizó, en primer lugar, una búsqueda de las principales herramientas existentes; en segundo lugar, se seleccionaron aquellas que medían de forma gratuita los items que considerábamos necesarios. Para Facebook se utilizó una única herramienta, Fanpage Karma, ya que mide casi todos los items propuestos (reacción por tipo de post, engagement, tiempo y ratio de respuesta, número de fans, me gusta, comentarios y comparticiones, número de posts y tipo de post):

http://www.fanpagekarma.com

Para analizar Twitter se utilizaron dos herramientas:
- Twitalyzer para medir el engagement, los seguidores más influyentes, la audiencia activa y el origen de estos seguidores: http://www.twitalyzer.com

- Hhotwi para medir los seguidores y los seguidos, los tweets favoritos y los top hashtags entre otros: http://www.whotwi.com

Con posterioridad se llevó a cabo un análisis manual de los top posts de Facebook de los destinos seleccionados publicados durante los últimos 3 meses para completar los resultados de algunas variables que las herramientas online de medición analizaban por un período de tiempo excesivamente corto como para obtener resultados significativos.

El estudio se realizó entre el 25 de julio y el 22 de octubre de 2013; y el posterior de Facebook en junio de 2014 y comprendió los top posts de los 3 meses anteriores.

Es más aconsejable fomentar la interacción y seguimiento con los posts publicados que generar un gran número de ellos

\section{Resultados}

\subsection{Contenido}

Lo primero que se analizó fue la frecuencia de publicación de los posts o tweets. Como se observa en la tabla 1, la media de posts por día en Facebook es superior en Madrid, seguida de Las Palmas, Barcelona y Santiago. Sevilla se encuentra en la última posición.

En cuanto al formato de los posts en Facebook, se observa claramente que la fotografía es el más utilizado en todos los destinos. Madrid destaca en su uso y también en el uso de links a otros espacios del territorio relacionados, con un número incluso superior al de fotografías. Pero el resto de destinos utilizan los links en mucha menor proporción y Sevilla ninguno. Cabe destacar que Madrid también publica bastantes vídeos, mientras que el resto de destinos prácticamente no los utilizan.

Con respecto al tipo de posts publicados en Facebook, se observó que se hicieron escasas preguntas a los usuarios en las cuentas analizadas. Por tanto, se desaprovecha un

Tabla 1. Facebook. Media de posts por día y número de posts según tipo

\begin{tabular}{|lccccc|}
\hline & Barcelona & $\begin{array}{c}\text { Santiago de } \\
\text { Compostela }\end{array}$ & Sevilla & Madrid & $\begin{array}{c}\text { Las Palmas } \\
\text { de G.C. }\end{array}$ \\
\hline Posts por día* & 1,4 & 0,7 & 0,2 & 2,1 & 1,7 \\
\hline Tipo de post** & & & & & \\
Estatus & 0 & 0 & 0 & 0 & 3 \\
Imagen/foto & 34 & 7 & 7 & 85 & 25 \\
Link & 4 & 3 & 0 & 93 & 12 \\
Vídeo & 1 & 0 & 1 & 11 & 2 \\
Ofertas & 0 & 0 & 0 & 0 & 0 \\
Música & 0 & 0 & 0 & 0 & 0 \\
Diapositivas & 0 & 0 & 0 & 0 & 0 \\
Otros & 0 & 0 & 0 & 0 & 0 \\
\hline
\end{tabular}

*media; **últimos 90 días 25/07/2013-22/10/2013 
Tabla 2. Twitter. № de días de existencia, $\mathrm{n}$ o de tweets del destino, $\mathrm{n}$ ㅇ de tweets de la audiencia, frecuencia de tweets del destino por día y tipo de tweet

\begin{tabular}{|lccccc|}
\hline & Barcelona & $\begin{array}{c}\text { Santiago de } \\
\text { Compostela }\end{array}$ & Sevilla & Madrid & $\begin{array}{c}\text { Las Palmas } \\
\text { de G.C. }\end{array}$ \\
\hline Días de existencia & 1.190 & 1.582 & 1.696 & 663 & 703 \\
No tweets destino & 1.645 & 8.548 & 6.791 & 4.315 & 7.978 \\
No tweets audiencia* & 2.063 & 1.425 & 989 & 1.906 & 1.946 \\
Frec. tweets por día* & 1,4 & 5,4 & 4,0 & 6,5 & 1,3 \\
Tipo de tweet **: & & & & & \\
URL (linked rate) & 49,1 & 44,4 & 48,2 & 27,2 & 11,7 \\
Reply (@rate) & 24,7 & 31,7 & 35,7 & 46,3 & 67,2 \\
RT (ReTweet) & 25,7 & 20,9 & 14,1 & 25,5 & 16,1 \\
Normal & 0,5 & 3,0 & 2,0 & 1,0 & 5,0 \\
\hline
\end{tabular}

*media; **porcentaje

potencial importante que posee esta red social para crear diálogo, ya que a través de las preguntas, que son una invitación directa al usuario a ofrecer una respuesta, se podría facilitar una mayor interacción.

En el caso de Twitter, los resultados son los que se muestran en la tabla 2.

Sevilla fue el primer destino que creó su Twitter oficial, seguido de Santiago de Compostela en 2009 y Barcelona en 2010. Sin embargo, y aunque el mayor número de tweets se acumula en la cuenta de Santiago de Compostela, destaca especialmente el elevadísimo número de tweets que posee Las Palmas en relación con el poco tiempo que lleva activo. Así, pese a ser el penúltimo destino en crear su cuenta, ya supera en número de tweets totales al destino de primera creación.

Si observamos en la tabla 2 el tipo de tweets publicados, aproximadamente la mitad de los de Barcelona, Santiago y Sevilla incluyen links a urls, un $30 \%$ son respuestas, un $20 \%$ son retweets y alrededor del $2 \%$ son tweets de estatus (estos últimos son simples comentarios, que pueden incluir elementos como fotografías, pero que no incluyen links ni menciones y no son respuestas). En cambio, en Las Palmas y Madrid se reduce considerablemente el porcentaje de tweets con link de url y se incrementa el de tweets de respuesta, lo que significa que estos destinos utilizan más Twitter para crear diálogo con los usuarios que para difundir únicamente información.

Reacciones medias por tipo de post

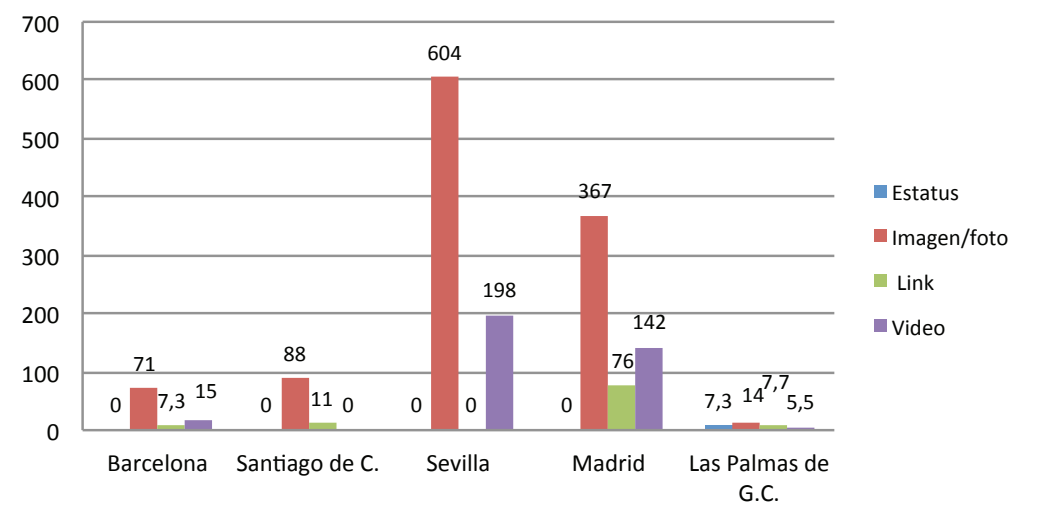

Gráfico1. Media de reacciones de los usuarios por tipo de post, publicados en los últimos 90 días
Respecto a la temática de los posts, en Facebook se midió a través de las top keywords o palabras clave más repetidas y en Twitter, a través de los hashtags más utilizados. Lo que se observa en común es que generalmente las palabras más repetidas son las del nombre o la marca del destino. Pero a pesar de que se repite el nombre de la marca, el resto de contenidos no tratan de comunicar su identidad y sus principales atributos. Por otro lado se observa que las herramientas de medición online utilizadas no aportan casi información sobre el contenido de los posts, lo que dificulta un análisis en profundidad de este aspecto.

\subsection{Interactividad}

En este parámetro, una de las principales variables que se midió en Facebook fue la reacción de los usuarios por tipo de post, que aporta resultados muy interesantes. Si tomamos los datos según el tipo de post y lo comparamos con las reacciones, obtenemos un índice de reacción. Resulta curioso ver que los posts con fotografías son los que reciben una mayor reacción por parte de los usuarios. Los vídeos también despiertan reacciones, aunque en menor proporción. Le siguen los links, que generan muy pocas reacciones y en último lugar están los posts de estatus, que no generan prácticamente ninguna reacción.

En el gráfico 1 se observa que el Facebook de Sevilla es el que genera más reacciones con sus fotografías, seguido del de Madrid. El resto tiene reacciones muy bajas.

Otro de los items analizados, muy relacionado con las reacciones, es el engagement (ver tabla 3), que mide las reacciones de los usuarios, los me gusta, los comentarios y las comparticiones.

Si comparamos el número medio de posts colgados por día y el engagement medio que generan se observa que no existe una correlación lógica. Así, aunque Madrid publica más posts, los de Las Palmas generan mayor engagement. Es decir, no por generar más posts se obtiene un nivel más elevado de compromiso o fidelidad hacia el sitio, por lo que es más aconsejable fomentar la interacción y seguimiento con los posts publicados que generar un gran número de posts al día con un bajo engagement.

En Twitter las herramientas de análisis no permitieron medir las reacciones por tipo de tweet, pero sí el engagement comparativamente con la frecuencia de tweets y el número de seguidores (tabla 4).

Se observa que Sevilla es el destino que recibe más menciones y más retweets, con gran diferencia del resto, a pesar de que su promedio de publicación de tweets por día es medio-bajo. 
No obstante cabe destacar especialmente la gestión de Las Palmas, que posee también un elevado número de menciones, aunque en este caso el dato sí se corresponde con la publicación de un elevado número de tweets por día, muy por encima del resto de destinos. Además es el destino que obtiene más tweets de la audiencia, también muy por encima del resto, incluso de Sevilla (véase tabla 2). Ello significa que su gestión es muy activa y que precisamente por eso consigue mucha participación. Pero además logra mucho engagement de sus públicos, tal vez, tal y como hemos apuntado anteriormente, porque es el destino que emite más respuestas y retweets, es decir, el destino que mejor utiliza Twitter para generar diálogo con sus públicos.

\subsection{Visibilidad}

En cuanto a este último parámetro, un índice clave de medición es el número de fans en Facebook o seguidores en Twitter, que muestra la visibilidad que tiene el sitio, y que todo destino turístico debería tratar de aumentar.

Los resultados muestran que las cuentas de Facebook de Sevilla son las que tienen más fans, seguidas de Madrid y Barcelona (tabla 5).

Otros índices interesantes por lo que respecta a la visibilidad en Facebook son la media de likes ("me gusta"), la media de comentarios y la media de comparticiones por post. Se observa que Sevilla posee índices muy superiores al resto. Es cierto que Sevilla tiene 1.000 fans más que Madrid, pero la diferencia en "me gusta", comentarios y comparticiones es muy superior a la diferencia del número de fans. Ello demuestra el éxito de los comentarios en Facebook de Sevilla. También cabe destacar la diferencia entre Santiago y Barcelona. Barcelona tiene 1.000 fans más que Santiago y en cambio sus comentarios generan casi el mismo número de "me gusta" y más número de comparticiones.

En cuanto a la visibilidad en Twitter, las herramientas de análisis ofrecen información sobre un mayor número de aspectos.

El índice más importante es el número de seguidores, comparable con el número de fans del Facebook. Los resultados (tabla 6) muestran que Sevilla posee muchísimos más seguidores que el resto de destinos, con una gran diferencia. Sevilla también destaca en otros índices como el Klout score, el impacto, el nivel de karma y la profile performance.

En cambio, si observamos el número de tweets marcados como favoritos, que sería una medición bastante parecida a los "me gusta" en Facebook, sorprende el bajo resultado de Sevilla, a pesar de su elevado número de seguidores. También sorprenden los elevados índices de Las Palmas a pesar de que es la cuenta de Twitter con menos seguidores, lo que
Tabla 3. Facebook. № de fans, media de posts por día del destino y engagement.

\begin{tabular}{|lrrrrc|}
\hline & Barcelona & $\begin{array}{r}\text { Santiago de } \\
\text { Compostela }\end{array}$ & Sevilla & Madrid & $\begin{array}{c}\text { Las Palmas } \\
\text { de G.C. }\end{array}$ \\
\hline No de fans* $^{*} 16.618$ & 4.769 & 47.039 & 35.182 & 1.456 \\
Posts por día* & 1,40 & 0,70 & 0,20 & 2,10 & 1,70 \\
Engagement $^{* *}$ & 0,34 & 1,16 & 0,27 & 1,27 & 2,11 \\
\hline
\end{tabular}

Tabla 4. Twitter. № de seguidores, media de tweets por día, engagement y engagement por tipo de tweet.

\begin{tabular}{|lccccc|}
\hline & Barcelona & $\begin{array}{c}\text { Santiago de } \\
\text { Compostela }\end{array}$ & Sevilla & Madrid & $\begin{array}{c}\text { Las Palmas } \\
\text { de G.C. }\end{array}$ \\
\hline No seguidores $^{\circ}$ & 3.587 & 7.337 & 34.417 & 4.566 & 1.891 \\
tweets por día* & 1,4 & 5,4 & 4,0 & 6,5 & 11,3 \\
Engagement total* $^{*}$ & 47 & 235 & 559 & 108 & 272 \\
Menciones** $^{*}$ & 31 & 87 & 221 & 52 & 101 \\
Retweets & 6 & 76 & 291 & 33 & 43 \\
Tweets enviados & 10 & 72 & 47 & 23 & 128 \\
\hline
\end{tabular}

*media (fecha de análisis: 22/10/2013).

**total de menciones de estas páginas de destinos en los perfiles de los usuarios (fecha de análisis: 22/10/2013)

Tabla 5. Facebook. № de fans, no de posts, media de "me gusta", media de comentarios media de comparticiones.

\begin{tabular}{|lccccc|}
\hline & Barcelona & $\begin{array}{c}\text { Santiago de } \\
\text { Compostela }\end{array}$ & Sevilla & Madrid & $\begin{array}{c}\text { Las Palmas } \\
\text { de G.C. }\end{array}$ \\
\hline No de fans* $^{*}$ & 16.618 & 4.769 & 47.039 & 35.182 & 1.456 \\
No de posts** $^{*}$ & 39 & 10 & 8 & 189 & 42 \\
likes (media)** & 54,18 & 54,71 & 432,50 & 176,37 & 9,66 \\
comentarios (media)** $^{*}$ & 1,59 & 1,54 & 18,75 & 4,38 & 0,38 \\
shares (media)** & 7,51 & 8,49 & 102,13 & 30,02 & 1,17 \\
\hline
\end{tabular}

*en el momento de análisis 22/10/2013; **últimos 90 días 25/07/2013-22/10/2013

Twitter. № seguidores, № seguidores de la audiencia, índice Klout, índice de impacto, índice karma, índice de performance del sitio y no de tweets que se han

\begin{tabular}{|lccccc|}
\hline & Barcelona & $\begin{array}{c}\text { Santiago de } \\
\text { Compostela }\end{array}$ & Sevilla & Madrid & $\begin{array}{c}\text { Las Palmas } \\
\text { de G.C. }\end{array}$ \\
\hline No seguidores & 3.587 & 7.337 & 34.417 & 4.566 & 1.891 \\
No seguidores audiencia* & 3.336 & 749 & 410 & 2.236 & 981 \\
Klout score & 53,7 & 60,4 & 68,7 & 59,1 & 54,6 \\
Impacto & 0,8 & 1,8 & 8,4 & 1,2 & 0,8 \\
Nivel de karma & 4,4 & 7,2 & 14 & 6,8 & 9,9 \\
Profile performance (\%) & 29 & 41 & 54 & 42 & 42 \\
Favourite times & 0 & 4 & 16 & 482 & 124 \\
\hline
\end{tabular}

*media (fecha de análisis: 22/10/2013)

implica que el éxito de los tweets de Las Palmas es superior al de los tweets de otros destinos.

\section{Conclusiones}

En primer lugar, los resultados muestran que la mayoría de posts en las cuentas de Facebook de los destinos poseen fotografías, aunque algunos, como Sevilla o Santiago, todavía las utilizan poco. También muestran que los posts con fotografías son los que consiguen más reacciones entre los usuarios o públicos, seguidos de aquellos que muestran vídeos. De ello se desprende, como primera recomendación, que es aconsejable un superior uso de las fotografías en las redes sociales de los destinos como mecanismo para generar interacción. 
Por otra parte, se observa que Facebook se utiliza fundamentalmente para difundir información, lo que unido a la ausencia de elementos que propicien la creación de diálogo, como las preguntas, refleja un escaso interés de los gestores por favorecer la interacción directa con los públicos. De este modo se desaprovecha el potencial interactivo y relacional de esta red.

En cambio, algunos de los destinos analizados sí utilizan Twitter para crear diálogo con sus públicos y los resultados muestran que precisamente estos destinos poseen un engagement más elevado. En este sentido se observa que los tweets de respuesta y retweets son importantes para crear diálogo con los públicos y, por tanto, su uso es muy recomendable.

Los posts con fotografías son los que consiguen más reacciones

Por otro lado se ha constatado que las herramientas de análisis online utilizadas poseen muchas limitaciones: no aportan información completa e interesante sobre la temática de los posts/tweets y miden muchos aspectos sin explicar cómo lo hacen. Por tanto se recomienda la creación de una metodología específica de análisis comunicativo de los medios sociales de los destinos turísticos.

Respecto a la comunicación de la marca de los destinos, podemos afirmar que, a pesar de que en el análisis de contenido predominan los nombres de las ciudades, no se comunica su identidad. Fundamentalmente los contenidos se centran en informaciones sobre ocio y atractivos turísticos y se descuida la comunicación de la marca del lugar.

En síntesis, se recomienda que los gestores de los medios sociales hagan más preguntas a sus usuarios y los estudien para conocer el tipo de público al que se dirigen (futuros turistas, ciudadanos del lugar que viven fuera, profesionales del turismo, instituciones, etc.), cuáles son sus intereses respecto a los medios sociales y su relación con el lugar, y que se dirijan a ellos con intención de crear diálogo y buenas relaciones. Igualmente se recomienda que utilicen los medios sociales para comunicar la marca y la identidad del destino y no sólo sus atractivos turísticos. Tan sólo así se aprovechará todo el potencial que ofrecen estas webs y ello se traducirá en el éxito comunicativo de los destinos.

Los tweets de respuesta y retweets son importantes para crear diálogo

\section{Notas}

1. Este estudio interdisciplinar de comunicación y turismo es parte del proyecto de investigación "Uso e influencia de los medios sociales y la comunicación 2.0 en la toma de decisiones turísticas y en la imagen de marca de los destinos. Aplicaciones de utilidad para los destinos turísticos españoles" (CSO2012-34824).

2. Las áreas Nielsen son zonas geográficas determinadas por la empresa de investigación de mercados Nielsen en función de sus características mercadológicas relativamente homogéneas, y por ser representativas de todo el territorio español.

\section{Bibliografía}

Agarwal, Shalabh; Mondal, Akash; Nath, Asoke (2011). "Social media - The new corporate playground". International journal of research and reviews in computer science (ljrrcs), v. 2, n. 3, pp. 696-700.

Ayeh, Julian K.; Au, Norman; Law, Rob (2013). "Predicting the intention to use consumer-generated media for travel planning". Tourism management, v. 35, pp. 132-143.

Berthon, Pierre R.; Pitt, Leyland F.; Plangger, Kirk; Shapiro, Daniel (2012). “Marketing meets Web 2.0, social media, and creative consumers: Implications for international marketing strategy". Business horizons, v. 55, pp. 261-271. http://dx.doi.org/10.1016/j.bushor.2012.01.007

Cox, Carmen; Burgess, Stephen; Sellitto, Carmine; Buultjens, Jeremy (2009). "The role of user-generated content in tourists' travel planning behavior". Journal of hospitality marketing \& management, v. 18, pp. 743-764.

http://dx.doi.org/10.1080/19368620903235753

Fotis, John; Buhalis, Dimitrios; Rossides, Nicos (2012). "Social media use and impact during the holiday travel planning process". En: Fuchs, M.; Ricci, F.; Cantoni, L. Information and communication technologies in tourism, Vienna, Austria: Springer-Verlag, pp. 13-24. ISBN: 9783709111413

Hays, Stephanie; Page, Stephen J.; Buhalis, Dimitrios (2013). "Social media as a destination marketing tool: its use by national tourism organisations". Current issues in tourism, v. 16, n. 3, pp. 211-239.

http://dx.doi.org/10.1080/13683500.2012.662215

Hvass, Kristian A.; Munar, Ana M. (2012). "The takeoff of social media in tourism". Journal of vacation marketing, $\mathrm{v}$. 18, n. 2, pp. 93-103.

http://dx.doi.org/10.1177/1356766711435978

InternetWorldStats. Internet usage statistics. http://www.internetworldstats.com/stats.htm

Jacobsen, Jens K. S.; Munar, Ana M. (2012). "Tourist information search and destination choice in a digital age". Tourism management perspectives, v. 1, pp. 39-47. http://dx.doi.org/10.1016/j.tmp.2011.12.005

Kaplan, Andreas M.; Haenlein, Michael (2010). “Users of the world, unite! The challenges and opportunities of social media". Business horizons, v. 53, pp. 59-68. http://dx.doi.org/10.1016/j.bushor.2009.09.003

Leung, Daniel; Law, Row; Van-Hoof, Hubher; Buhalis, Dimitrios (2013). "Social media in tourism and hospitality: A literature review". Journal of travel \& tourism marketing, v. 30, n. 1, pp. 3-22. http://dx.doi.org/10.1080/10548408.2013.750919

Litvin, Stephen W.; Goldsmith, Ronald E.; Pan, Bing (2008). "Electronic word-of-mouth in hospitality and tourism management". Tourism management, v. 29, n. 3, pp. 458-468.

http://dx.doi.org/10.1016/j.tourman.2007.05.011 
Munar, Ana M. (2011). "Tourist-created content: rethinking destination branding". International journal of culture, tourism and hospitality research, v. 5, n. 3, pp. 291-305.

http://dx.doi.org/10.1108/17506181111156989

Murphy, Laurie; Moscardo, Gianna; Benckendorff, Pierre (2007). "Using brand personality to differentiate regional tourism destinations". Journal of travel research, v. 46, n. 1, pp. 5-14. http://dx.doi.org/10.1177/0047287507302371

Pring, Cara (2012). Social media and internet statistics.

http://thesocialskinny.com/216-social-media-and-internetstatistics-september-2012

Sigala, Marianna (2009). "Web 2.0, social marketing strategies and distribution channels for city destinations: enhancing the participatory role of travelers and exploiting their collective intelligence". En: Gascó-Hernández, M.; TorresCoronas, T. Information communication technologies and city marketing. Digital opportunities for cities around the world. Hershey: Information Science Reference. ISBN: 978 1605669823

http://dx.doi.org/10.4018/978-1-60566-982-3.ch068

World Travel Market. "World travel market 2010 industry report".

http://www.wtmlondon.com/files/wtm2010_industry_ report.pdf

Xiang, Zheng; Gretzel, Ulrike (2010). "Role of social media in online travel information search". Tourism management, v. 31, pp. 179-188.

http://dx.doi.org/10.1016/j.tourman.2009.02.016

\section{Inforảrea}

\section{Ayudamos a tu organización en la transformación digital y el gobierno de la información}

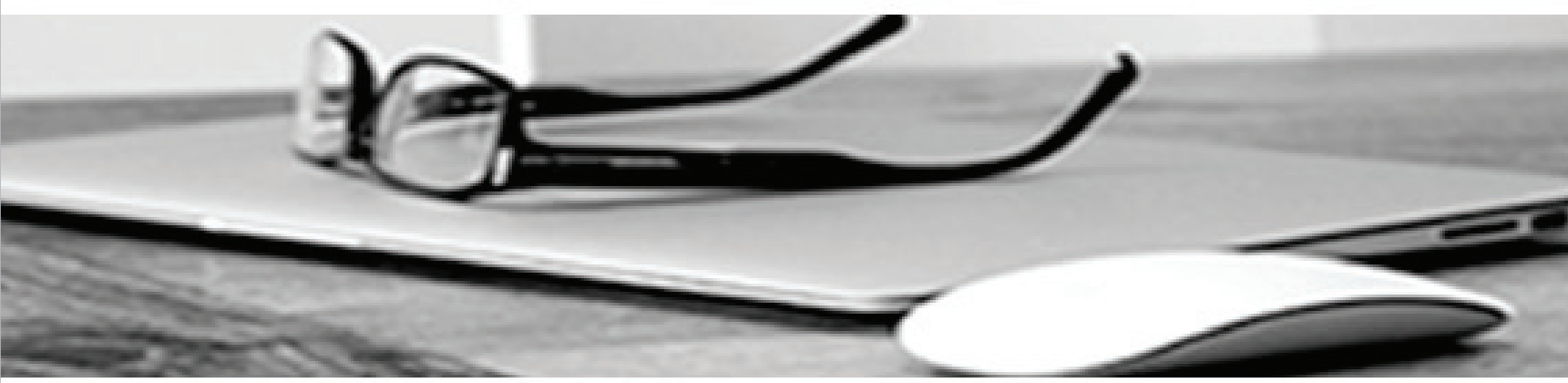

* Consultoría estratégica en gestión y gobierno de la información

* Gestión documental y "records management"

* Gestión de contenidos, intranets corporativas y entornos de colaboración

* Estudios especializados

Clientes satisfechos, cientos de empresas nacionales e internacionales y más de 30 años de experiencia son la mejor garantía de nuestra reputación. 